\title{
ERRATUM
}

\section{The Histone Chaperone Anti-Silencing Function 1 Is a Global Regulator of Transcription Independent of Passage through S Phase}

Susan R. Zabaronick and Jessica K. Tyler

Department of Biochemistry and Molecular Genetics, School of Medicine, University of Colorado, Aurora, Colorado

Volume 25, no. 2, p. 652-660, 2005. Page 652, top: "Received 1 October 2003" should read "Received 1 October 2004." 